\title{
Time-Resolved Fluorescence Spectroscopy Study of Energy Transfer Dynamics in Phycobilisomes from Cyanobacteria Thermosynechococcus vulcanus NIES 2134 and Synechocystis sp. PCC 6803
}

\author{
Mingyuan Xie ${ }^{1,2,+} \mathbb{D}$, Wenjun $\mathrm{Li}^{3,4,+}$, Chao Xiao ${ }^{1,+}$, Zhanghe Zhen ${ }^{3}$, Jianfei Ma ${ }^{5}$, Hanzhi Lin ${ }^{6}\left(\mathbb{D}\right.$, Song Qin ${ }^{3,4, *(\mathbb{D})}$ \\ and Fuli Zhao ${ }^{1, *}$
}

check for updates

Citation: Xie, M.; Li, W.; Xiao, C.; Zhen, Z.; Ma, J.; Lin, H.; Qin, S.; Zhao, F. Time-Resolved Fluorescence Spectroscopy Study of Energy Transfer Dynamics in Phycobilisomes from Cyanobacteria Thermosynechococcus vulcanus NIES 2134 and Synechocystis sp. PCC 6803. Crystals 2021, 11, 1233. https://doi.org/10.3390/ cryst11101233

\section{Academic Editors:}

Jesús Sanmartín-Matalobos, Ana

M. Garcia-Deibe and

Alessandro Vergara

Received: 27 August 2021

Accepted: 12 October 2021

Published: 13 October 2021

Publisher's Note: MDPI stays neutral with regard to jurisdictional claims in published maps and institutional affiliations.

Copyright: (c) 2021 by the authors. Licensee MDPI, Basel, Switzerland. This article is an open access article distributed under the terms and conditions of the Creative Commons Attribution (CC BY) license (https:// creativecommons.org/licenses/by/ $4.0 /)$.
1 State Key Laboratory of Optoelectronic Materials and Technologies, School of Physics, Sun Yat-Sen University, Guangzhou 510275, China; xiemy5@mail.sysu.edu.cn (M.X.); xiaoch23@mail2.sysu.edu.cn (C.X.)

2 Institute of Advanced Science Facilities, Shenzhen 518107, China

3 Yantai Institute of Coastal Zone Research, Chinese Academy of Sciences, Yantai 264003, China; wjli@yic.ac.cn (W.L.); zhzhen@yic.ac.cn (Z.Z.)

4 Center for Ocean Mega-Science, Chinese Academy of Sciences, Qingdao 266071, China

5 State Key Laboratory of Membrane Biology, Beijing Advanced Innovation Center for Structural Biology, School of Life Sciences, Tsinghua University, Beijing 100084, China; sddymjf@126.com

6 Institute of Marine and Environmental Technology, University of Maryland Center for Environmental Science, Baltimore, MD 21202, USA; youralfred@gmail.com

* Correspondence: sqin@yic.ac.cn (S.Q.); stszfl@mail.sysu.edu.cn (F.Z.)

$\dagger \quad$ These authors contribute equally.

Abstract: As the largest light-harvesting complex in cyanobacteria, phycobilisomes (PBSs) show high efficiency and a high rate of energy transfer, owing to an elegant antenna-like assembly. To understand the structural influence on the dynamic process of the energy transfer in PBSs, two cyanobacterium species Thermosynechococcus vulcanus NIES 2134 (T. 2134) and Synechocystis sp. PCC 6803 (S. 6803) with different rod-core-linked assemblies were chosen for this study. The dynamic process of the energy transfer in both PBSs was investigated through time-resolved fluorescence spectroscopy (TRFS) with a time resolution of sub-picosecond. Via the fluorescence decay curves deconvolution, the pathways and related rates of the excitation energy transfer (EET) were determined. Three time components, i.e., 10, 80, and 1250 ps, were identified in the EET in the PBSs of T. 2134 and three, i.e., 9, 115, and 1680 ps, in the EET in the PBSs of S. 6803. In addition, a comparison of the dynamic process of the energy transfer between the two cyanobacteria revealed how the PBS assembly affects the energy transfer in PBSs. The findings will provide insight into future time-resolved crystallography.

Keywords: phycobilisomes; cyanobacteria; time-resolved fluorescence spectroscopy; energy transfer

\section{Introduction}

Cyanobacteria are the most ecologically important phototrophic bacteria on earth. They constitute a phylogenetically coherent group of morphologically and evolutionarily ancient diverse bacteria [1]. Cyanobacteria are characterized by their capability to photosynthesize. They synthesize PBSs as light-harvesting antennas composed of proteins that binding with various pigments. The PBSs exhibit extremely efficient light absorption and energy transfer properties [2]. Previous research has discovered the relationship between the intricate structural and functional organization of PBSs from cyanobacteria and their high energy transfer efficiency [3-6].

The geometry of a PBS molecule in cyanobacteria is an elegant antenna-like assembly, which is made up of phycobiliproteins (PBPs). A PBS molecule has a centrally located core composed of two to five allophycocyanin (APC), and in most cases surrounded by six to 
eight phycocyanin $(\mathrm{PC})$ rods $[7,8]$. The mega architecture involves additional non-pigmentbinding proteins called linker proteins (LP) [9], which are critical proteins for determining the directionality of excitation energy transfer [7]. The PBPs are assembled from subunits $\alpha$, $\beta$. An $\alpha$ and $\beta$ subunit combine into a monomer $(\alpha \beta)$. A PC monomer involves three types of phycocyanobilin (PCB): $\alpha 84, \beta 84$, and $\beta 155$ [10-12], while an APC monomer involves only two types: $\beta 84$ and $\alpha 84$ [13-16]. The monomers then assemble into trimers, hexamers, and further rods or cores, and finally form a complete PBS molecule.

Based on Förster resonance energy transfer (FRET) theory, researchers have proposed different mechanisms of excitation energy transfer (EET) between sub-complexes in cyanobacteria PBS. The chosen energy transfer pathways and energy transfer rate were determined by the relative distance between nearby chromophores and orientations of the dipole moments. Womick et al. showed a fast sub-picosecond EET component of $970 \mathrm{fs}$ in the PC hexamer [17], Zhang et al. found a time component of 18 ps which described an EET pathway from a terminal PC trimer to the APC core [18]. Recently, Hirota et al. demonstrated the EET from the rod to the core and finally to the terminal emitter, taking place in a timescale from several ten picosecond seconds to nanoseconds within the PBS of Thermosynechococcus vulcanus by time-resolved fluorescence and pump-probe technique [19].

In most of the determined PC structures in cyanobacteria PBSs, the rod-like structures are hexamer-hexamer interactions. However, the thermophilic cyanobacterium T. 2134, consisting of three APC core cylinders surrounded by six PC rods, involves a special PC trimer $\left(\mathrm{PC}_{612}\right)$ subunit that has been identified to date only in T. 2134 [20]. The special PC trimers locate at the terminal of the rods and fill the gap between the PC rods and the APC cores (Figure 1). Without forming $(\alpha \beta)_{6}$ hexamers, the $\alpha$ subunit in the monomer of the special $\mathrm{PC}_{612}$ possesses a higher degree of flexibility. Moreover, the fact of $\mathrm{PC}_{612}$ isolation in conjunction with APC cores demonstrates the strong connection between the $\mathrm{PC}_{612}$ and the APC cores. As a result, the performance of energy transfer dynamics in T. 2134 PBS may alter due to the special PC trimer, especially the dynamics of the energy transfer between the PC rods and the APC cores. Although scientists have resolved the individual PBPs structures via crystallography $[9,21,22]$, to the best of our knowledge, the T. 2134 PBS with complete structure has not been published. Thus, how the PBS assembly affects the energy transfer dynamics remains a mystery, and the structural foundations of energy transfer are still not well understood.

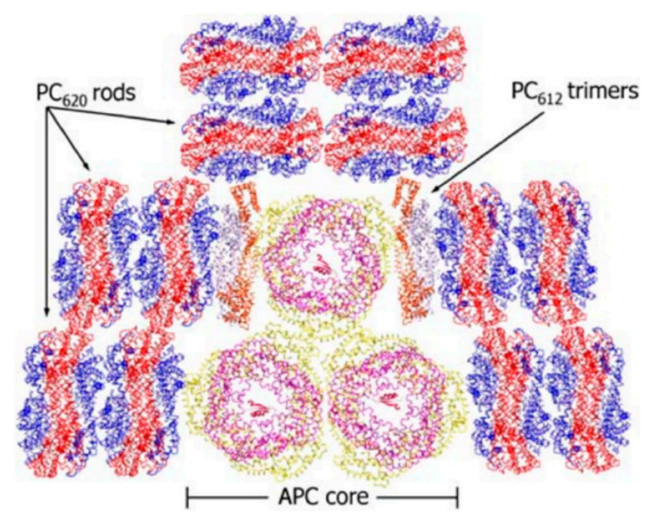

Figure 1. Architecture diagram of the rod-core assembly of PBSs from T. 2134 [23].

In this study, the PBS from T. 2134 with a special rod-core link structure was selected to investigate the structural induced energy transfer mechanism. Moreover, the PBS from S. 6803 with a similar PBS structure to that from T. 2134 was selected for comparison. The S. 6803 PBS is made up of three APC core cylinders surrounded by six PC rods, each consisting of three hexamers. The sequential energy flows in PBSs were provided by measuring both the steady-state and TRFS from T. 2134 and S. 6803 PBSs. The fluorescence decay curves at different emission wavelengths were deconvoluted for estimating the 
energy transfer components. In order to explore the structural basis of the energy transfer mechanism in a cyanobacteria PBS antenna, the differences between the energy transfer kinetics of the two cyanobacteria induced by the rod-core assembly were analyzed in detail.

\section{Materials and Methods}

\subsection{Growth of Cyanobacteria and Isolation of PBSs}

S. 6803 and T. 2134 were cultured at $22^{\circ} \mathrm{C}$ constant low light illumination (40 w white tubes and light cycle $16 \mathrm{~h}$, dark cycle $8 \mathrm{~h}$ ) in BG11 medium. Contamination was inhibited by filtering the medium with $0.22 \mu \mathrm{m}$ paper filters and the air supply with a cotton filter. PBSs from S. 6803 and T. 2134 were prepared according to the methods described in our previous studies [24] with modifications as follows: $0.75 \mathrm{~mol} \cdot \mathrm{L}-1 \mathrm{NaH}_{2} \mathrm{PO}_{4}-\mathrm{KH}_{2} \mathrm{PO}_{4}$ buffer, PH 7.0 were added to cyanobacteria pellets, and then Phenylmethanesulfonyl fluoride (PMSF) were used as protease inhibitor on PBSs isolation. Shedding light is ensured for every step.

\subsection{Detection of Steady-State and TRFS}

A spectrophotometer (TU-1810, Persee) was used to record the absorption spectra, A spectrofluorometer (FL-4500, Hitachi) was used to measure the steady-state fluorescence spectra, and the radiation profile of a standard lamp was used to correct the spectral sensitivity of the fluorometer. The experiments were performed at room temperature (298 K).

A polychromator coupled synchroscan streak camera (C6860, Hamamatsu, Japan), with a time resolution of $700 \mathrm{fs}$, was used to measure the TRFS at $77 \mathrm{~K}$, as shown in Figure 2. The PBSs samples were placed in a silica cuvette in the dark, frozen in liquid nitrogen. Glycerol was added to the PBSs samples as a cryoprotectant for $77 \mathrm{~K}$ spectral experiments. The pump source was a regenerative Ti:sapphire amplifier system (Legend Elite USP HE+, Coherent, Inc., Santa Clara, CA, USA), with output pulse laser at the wavelength of $570 \mathrm{~nm}$, which brought about excitation of PBSs. The pulse duration is $35 \mathrm{fs}$ and the pulse repetition rate is $1 \mathrm{kHz}$.

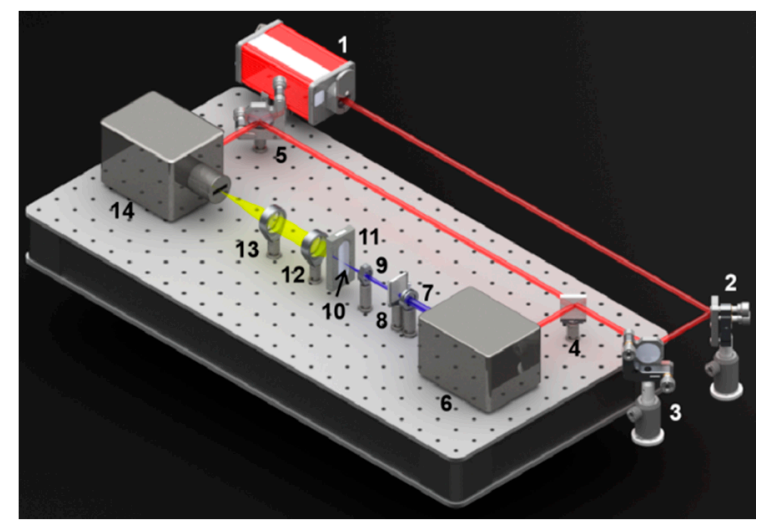

Figure 2. Experimental setup for time-resolved spectra measurement. 1: fs Amplifier system; 2,3,5: mirror; 4: beam splitter; 6: OPA; 7: aperture; 8: attenuator; 9,12,13: lens; 10: cuvette; 11: cryostat; 14: streak camera.

\subsection{Analysis of the TRFS}

With the temporal mode, we can obtain the fluorescence decay curves of PBSs. Then, we use a global optimization method to deconvolute the fluorescence decay curves [25]. The same lifetime parameters were used to analyze all the decay curves. The time resolution of fluorescence decay curves was approximately 2 ps after the deconvolution process. The FDAS was then provided by the amplitudes of the exponential components of the deconvoluted fluorescence decay curves as a function of emission wavelength [26]. 
The measured fluorescence was deconvolution after measurement by streak camera $[27,28]$. The measured fluorescence intensity $\mathrm{F}$ is described as:

$$
F(t)=f \otimes N=\int f(t) N\left(t-t^{\prime}\right) \mathrm{dt} t^{\prime}
$$

where $\mathrm{f}$ is the pump laser pulse. The theoretical fluorescence intensity $\mathrm{N}$ was considered with the form of a sum of multi-exponential:

$$
N(t)=\sum \varepsilon_{i} \exp \left(-t / \tau_{i}\right)
$$

On the basis of a global optimization algorithm, the deconvolution procedure was then applied to fit the isotropic fluorescence [26]. Here, $\tau_{i}$ represents the fluorescence isotropic decay constant, and A denotes the amplitude of $\tau_{i}$ :

$$
I_{n}(\lambda, \mathrm{t})=\sum_{i=1}^{n} A_{i}\left(\lambda_{i}\right) \exp \left(-t / \tau_{i}\right)
$$

\section{Results}

\subsection{Steady-State and Transient-State Spectroscopy Results}

The steady-state results of the two cyanobacteria PBSs at room temperature were obtained and analyzed in detail, as shown in Figure 3. For the PBS from S. 6803, its absorption spectrum at room temperature exhibited a peak at $621 \mathrm{~nm}$ and with a shoulder at $573 \mathrm{~nm}$, both of which were attributed to the absorption of PCB chromophores. In contrast, for the PBS from T. 2134, its PCB exhibited a peak at $621 \mathrm{~nm}$ and with a shoulder at $570 \mathrm{~nm}$, which was slightly different from those of the PBS from S. 6803. Nevertheless, the absorption peaks of $\mathrm{PC}_{612}$ were not detected via absorption spectroscopy inspection of a complete PBS. The fluorescence spectra of the two cyanobacteria PBSs were obtained upon excitation at $570 \mathrm{~nm}$. Specifically, the PBS from S. 6803 exhibited a fluorescence peak at $667 \mathrm{~nm}$, while the peak of the T. 2134 PBS was at $661 \mathrm{~nm}$. The integrity of cyanobacterial PBS is testified by Figure 3, that terminal emitter $667 \mathrm{~nm}$ is the main fluorescence emission peak of isolated PBSs.

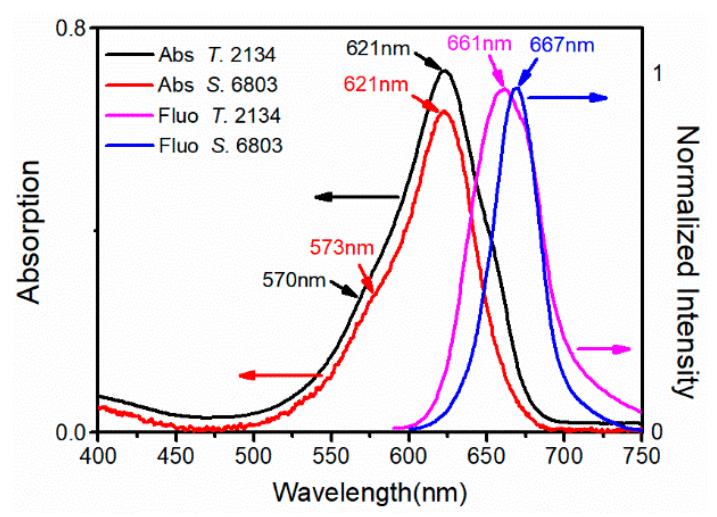

Figure 3. Absorption and fluorescence spectra of PBSs from S. 6803 and T. 2134 at room temperature.

\subsection{Time-Resolved Spectroscopy Results}

The steady-state fluorescence spectra combine the emissions from all fluorescence components, which cannot provide the energy transfer kinetics among them. Thus, the TRFS of the PBSs from the two cyanobacteria were measured at $77 \mathrm{~K}$ and analyzed in detail. At the temperature of $77 \mathrm{~K}$, molecular vibration, environmental influence such as solvent effect could be neglected, resulting in the precise reflection of energy transfer. This allows large signal-to-noise ratios and makes sure that the TRFS results are close to the assumptions of theoretical models for EET [29]. 
Figure 4 describes the TFRS of the PBSs from S. 6803 and T. 2134 at $77 \mathrm{~K}$ as color gradations. The color profiles represent the fluorescence intensity recorded at various delay times after excitation. Such representation helps us to understand the energy flow in a more conceptual way. The spectral changes in both PBSs are featured by the relative intensity of fluorescence components. At $570 \mathrm{~nm}$, the excitation wavelength for PCB chromophores, the TRFS of both PBSs showed an initial spike of the PC fluorescence band at $640-660 \mathrm{~nm}$, followed by the APC emission band around $680 \mathrm{~nm}$. The APC band rose as PC emission decayed. Moreover, compared with the PBS of S. 6803, the PC and APC bands of the T. 2134 PBS showed less overlap, which means less chromophore fluorescence leaking during the energy transfer process between the rod and the core. The result may indicate that the energy transfer process between the rod and the core is modulated by $\mathrm{PC}_{612}$, which has strong interactions with the APC core. The changes of the relative fluorescence components intensities reflect the EET from PC to APC in both PBSs.

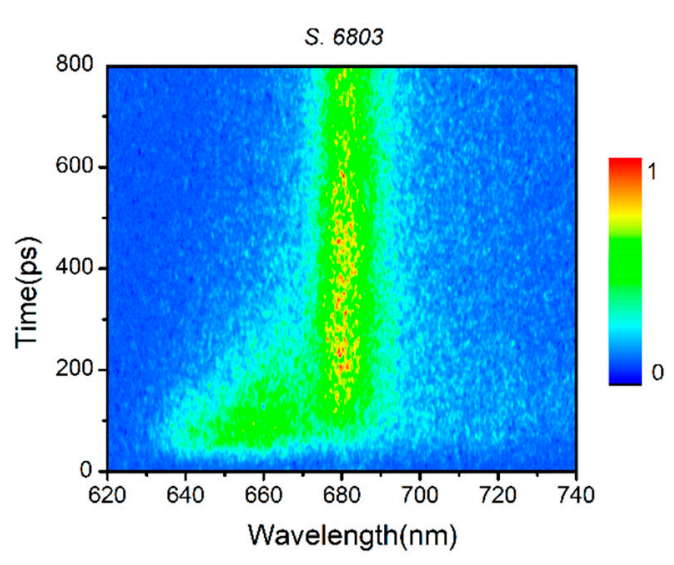

(a)

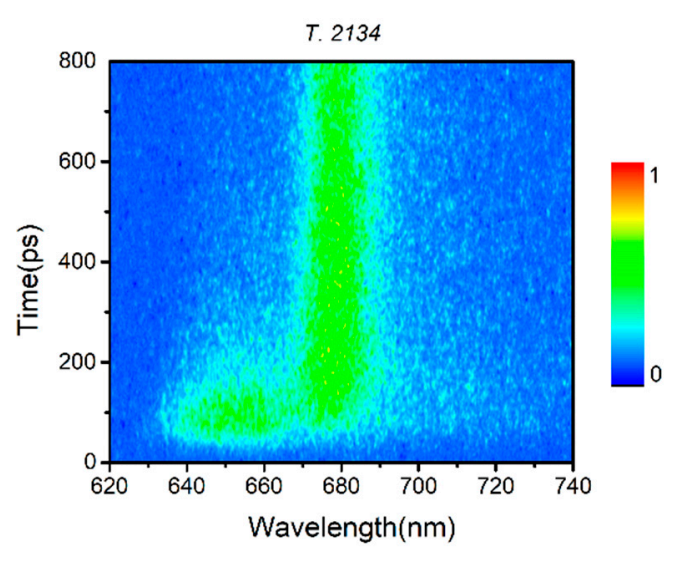

(b)

Figure 4. Time-resolved fluorescence spectra of the PBSs from (a) S. 6803 and (b) T. 2134 at 77 K. Excitation was set at $570 \mathrm{~nm}$.

Changes in the fluorescence spectra could be observed much more clearly in Figure 5. The synchroscan technology was used and the time-resolved fluorescence was obtained. The PC band emissions at 640-660 nm were detected in the frequency domain of the spectra of both PBSs. For the PBS from S. 6803, the PC emission band was the dominating band. Its peak was located at around $660 \mathrm{~nm}$ initially and then slowly exhibited a redshift. In contrast, the APC emission band around $680 \mathrm{~nm}$ rose gradually, growing higher than the PC band after $120 \mathrm{ps}$. Next, the APC emission took precedence, and the PC band gradually decreased and finally disappeared after $600 \mathrm{ps}$, suggesting that the rods converted all the absorbed solar energy to the core. For the PBS from T. 2134, its PC emission was also the dominating emission at the beginning. Then, the APC band rose as the PC emission decayed. The intensity of PC emission became relatively low after 280 ps and disappeared after 500 ps, indicating that the EET process from rod to core in the PBS from T. 2134 is faster than that in the PBS from S. 6803. 


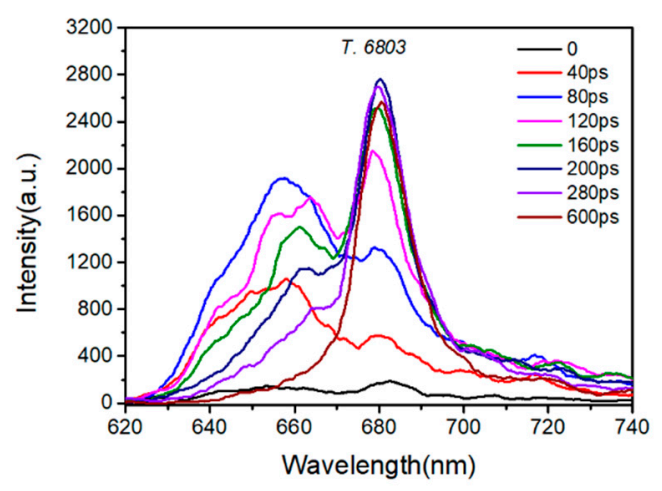

(a)

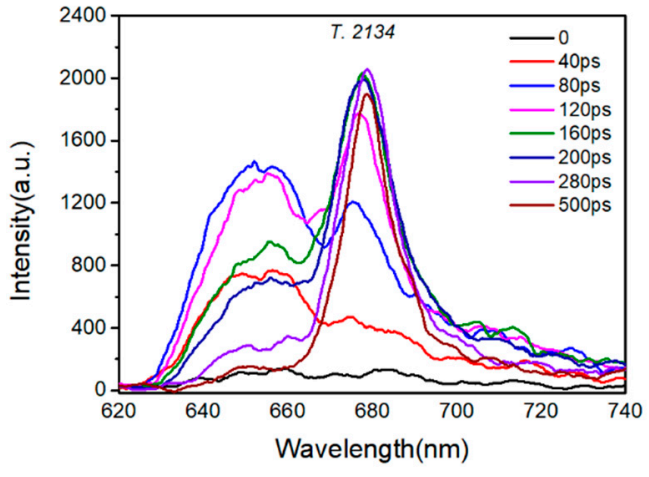

(b)

Figure 5. Evolution of the time-resolved fluorescence spectra of (a) S. 6803 and (b) T. 2134 at $77 \mathrm{~K}$. The spectra were obtained at the time of 40,80,120,160, 200, 280, 500, and 600 ps, respectively, after excitation. Excitation was set at $570 \mathrm{~nm}$.

\section{Discussion}

The deconvolution of time-resolved fluorescence decays curves was conducted to determine the fluorescence decay constants of the PBSs, adopting the Monte Carlo method for the experimental data process. The fluorescence intensity decay curves at the emission wavelength of 640,650,660,670,680, and $690 \mathrm{~nm}$ were selected for multi-exponential deconvolution. The fitting results of both PBSs were shown in Figure S1 (see Supplementary Materials). Here, the fluorescence intensity decay curves at the emission wavelengths of 660, 670, and $690 \mathrm{~nm}$ were selected for comparison, as shown in Figure 6.

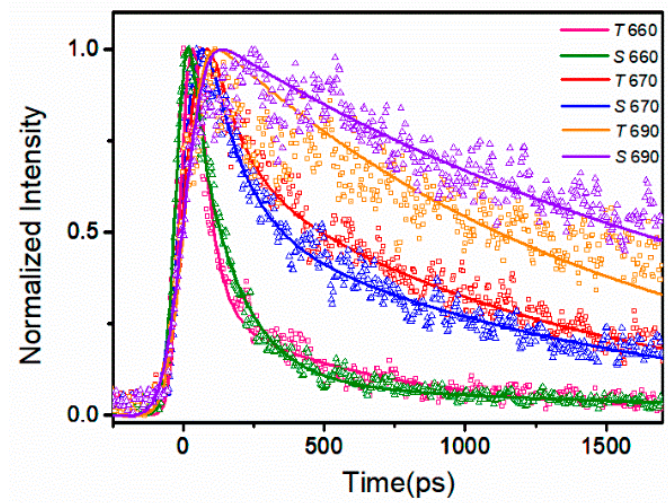

Figure 6. Fluorescence intensity decay curves and fitting results of PBSs from two PBSs at $77 \mathrm{~K}$. The detection wavelength was set at 660,670,690 nm. Excitation was set at $570 \mathrm{~nm}$. Triangles represent the experimental data of $S$. 6803, squares represent the experimental data of $T$. 2134, and colored lines represent the fitting results (labeled as S 660 with green line, S 670 with blue line, S 690 with violet line, T 660 with pink line, T 670 with red line, T 690 with orange line, respectively).

Tables 1 and 2 list the three time components $\tau$ and correspondent amplitudes $\mathrm{A}$ at different emission wavelengths. The fluorescence decay of the PBS from S. 6803 was found to fit the three exponentials of 9, 115, and $1680 \mathrm{ps}$ well, and the fluorescence decay of the PBS from T. 2134 fitted the three exponentials of 10, 80, and 1250 ps well. The two short time constants describe the time constant of the EET process, while the longest one timeconstants can be determined as the terminal fluorescent emission lifetime. The negative amplitudes suggest their corresponding components are the energy acceptors engaged in the uprising stage of fluorescence; otherwise, the components are energy donors engaged in the downturn stage of fluorescence. 
Table 1. Deconvolution results for S. 6803. Excitation was set at $570 \mathrm{~nm}$.

\begin{tabular}{ccccccc}
\hline $\mathbf{E}_{\mathbf{e m}} / \mathbf{n m}$ & $\boldsymbol{\tau}_{\mathbf{1}} / \mathbf{p s}$ & $\mathbf{A}_{\mathbf{1}} / \mathbf{\%}$ & $\boldsymbol{\tau}_{\mathbf{2}} / \mathbf{p s}$ & $\mathbf{A}_{\mathbf{2}} / \%$ & $\boldsymbol{\tau}_{\mathbf{3}} / \mathbf{p s}$ & $\mathbf{A}_{\mathbf{3}} / \%$ \\
\hline 640 & 12 & 80 & 93 & 18 & 1692 & 2 \\
650 & 11 & 82 & 127 & 16 & 1540 & 2 \\
660 & 9 & 73 & 150 & 25 & 1613 & 2 \\
670 & 8 & -4 & 120 & 62 & 1291 & 37 \\
680 & 9 & -1 & 116 & -10 & 1869 & 100 \\
690 & 8 & -3 & 88 & -1 & 2088 & 100 \\
Average & 9 & & 115 & & 1680 & \\
\hline
\end{tabular}

Table 2. Deconvolution results for T. 2134. Excitation was set at $570 \mathrm{~nm}$.

\begin{tabular}{ccccccc}
\hline $\mathbf{E}_{\mathbf{e m}} / \mathbf{n m}$ & $\boldsymbol{\tau}_{\mathbf{1}} / \mathbf{p s}$ & $\mathbf{A}_{\mathbf{1}} / \boldsymbol{\%}$ & $\boldsymbol{\tau}_{\mathbf{2}} / \mathbf{p s}$ & $\mathbf{A}_{\mathbf{2}} / \%$ & $\boldsymbol{\tau}_{\mathbf{3}} / \mathbf{p s}$ & $\mathbf{A}_{\mathbf{3}} / \%$ \\
\hline 640 & 14 & 48 & 76 & 48 & 1099 & 4 \\
650 & 10 & 70 & 101 & 26 & 1123 & 3 \\
660 & 9 & -8 & 63 & 88 & 929 & 11 \\
670 & 11 & -63 & 104 & 54 & 1218 & 46 \\
680 & 10 & -90 & 76 & 4 & 1744 & 97 \\
690 & 8 & -5 & 66 & -3 & 1369 & 100 \\
Average & 10 & & 80 & & 1250 & \\
\hline
\end{tabular}

Based on the deconvolution results, FDAS were obtained to reveal the dynamics of the energy transfer process in PBSs. In the FDAS, the positive factors indicate the kinetic fall of the excited populations, while the negative factors indicate the kinetic rise of the excited populations. The positive and negative bands coupling in the FDAS is a clear demonstration of the energy transfer from the positive-band pigments to the negative-band ones. Figure 7 shows the FDAS of the PBSs both from S. 6803 and T. 2134, and the decay constants are identical to the exponentials calculated by deconvolution for both PBSs.

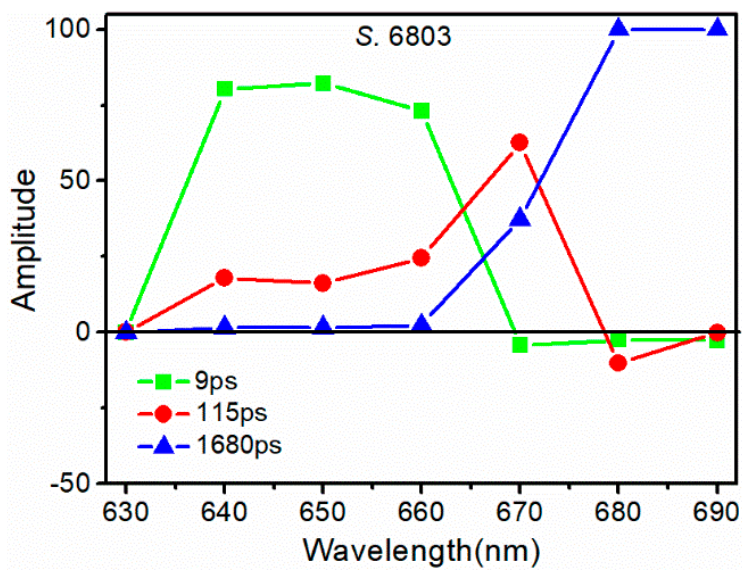

(a)

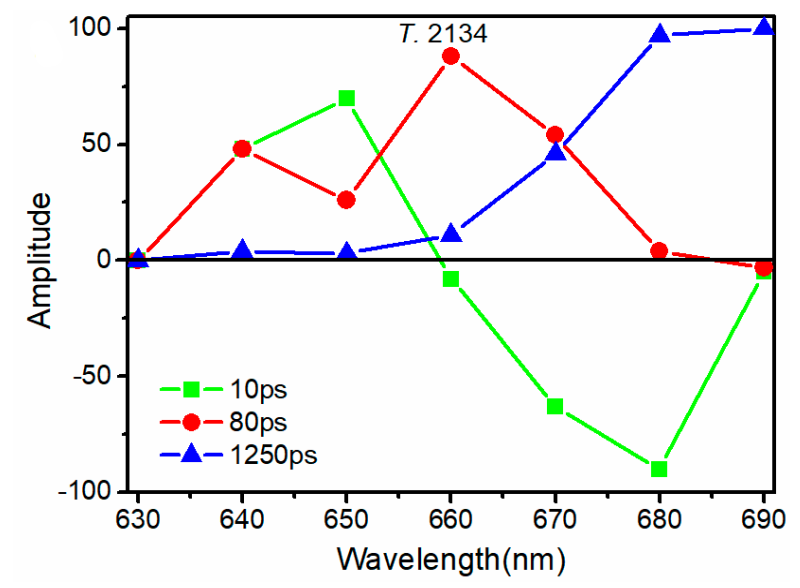

(b)

Figure 7. Fluorescence decay-associated spectra of the PBSs from (a) S. 6803 and (b) T. 2134.

For the PBS from S. 6803, the time component of 9 ps reflects the EET in the PC rod. Because at the blue side, the amplitude of FDAS is positive with a maximum around $650 \mathrm{~nm}$, while at the red side with no negative value (Figure 7a). Recently, Hirota et al. also identified an EET pathway with a time of 7.3 ps in the PC rod [19]. The time component of 115 ps could be assigned to the time of EET from the PC rod to the APC core. Because at the blue side, the amplitude of the FDAS was positive with a maximum around $670 \mathrm{~nm}$, then quickly turned negative at the red side with the minimum around $680 \mathrm{~nm}$. Based on the fluorescence spectra of the $S .6803 \mathrm{PBSs}$, it is reasonable to assign a time component of $115 \mathrm{ps}$ to the time of EET between the PC rod and APC core. Similarly, Holzwarth et al. [30] found 
an EET pathway from a PC rod to APC within 80-120 ps, depending on the rod length. The time component of 1680 ps reflects the fluorescence lifetime of the terminal emitter.

For the PBS from T. 2134, the time component of 10 ps was due to the transfer time of the pathways from $\mathrm{PC}_{612}$ to the APC core. Because at the blue side, the amplitude of the FDAS is positive with a maximum around $650 \mathrm{~nm}$, while at the red side, the amplitude turns to be negative with a minimum around $680 \mathrm{~nm}$ (Figure $7 \mathrm{~b}$ ). According to the fluorescence spectra of $\mathrm{PC}_{612}$ (Figure S2), it is reasonable to assign the time component of $10 \mathrm{ps}$ to the time of EET from $\mathrm{PC}_{612}$ and APC. Next, the time component of 80 ps could be assigned to the time of EET from the PC rod to the APC core. Because at the red side, the amplitude of the FDAS is positive with a maximum around $660 \mathrm{~nm}$, then turns to be negative at $690 \mathrm{~nm}$. The energy transfer time of 80 ps between the rod and the core is faster than that in the PBS from $S$. 6803. We believe that the special $\mathrm{PC}_{612}$ trimer, with strong conjunction to the APC core, speeds up the energy transfer rate between the rod and the core. The time component of 1250 ps reflects the fluorescence lifetime of the terminal emitter.

According to the results, a fast energy transfer rate of $10 \mathrm{ps}$ from the special $\mathrm{PC}_{612}$ trimer to the APC core was identified in the PBS of T. 2134. This unambiguously shows that the function of energy transfer is affected by the structure and assembly of the PBS. Compared to the PBS of $S .6803$ with a similar structure, the strong connection between the PC612 trimer and the APC core within the PBS of T. 2134 build a new energy transfer pathway that enables a faster energy transfer from the rod to the core.

\section{Conclusions}

We investigated the dynamic process of EET among PBPs in the PBSs from two cyanobacteria species by using femtosecond FRTS and deconvolution method, giving the energy transfer pathways and the energy transfer time constants. The results indicate that differences in the rod-core assemblies can significantly affect the rod-core energy transfer dynamics in PBSs. Furthermore, the strong conjunction between PC $_{612}$ trimers and the APC core leads to the fast energy transfer dynamics from the former to the latter. Moreover, the special $\mathrm{PC}_{612}$ trimer serves as a bridge to accelerate the energy transfer from the PC rods to the APC cores, which enables faster energy transfer from the rods to the cores. Furthermore, the energy transfer dynamics in the core are also altered by the trimer, ensuring that more energy is converted faster to PSII with less APC fluorescence emissions. The results of energy transfer dynamics from time-resolved PBSs spectroscopy will provide insight into future time-resolved crystallography.

Supplementary Materials: The following is available online at https:/ /www.mdpi.com/article/10.3 390/cryst11101233/s1. Figure S1: 77 K fluorescence intensity decay curves and fitting results of PBSs from S. 6803 and T. 2134. Figure S2: 77 K fluorescence emission spectrum of the PBS from T. 2134.

Author Contributions: Project supervision, S.Q. and F.Z.; preparation of samples, W.L., Z.Z. and J.M., steady-state microscopy data collection and analysis, W.L. and M.X.; time-resolved spectroscopy measurement, M.X. and C.X.; analysis of spectroscopy results, M.X., W.L. and H.L.; writing-original draft preparation, M.X. and W.L.; writing-review and editing, F.Z. and S.Q.; funding acquisition, F.Z., M.X. and S.Q. All authors have read and agreed to the published version of the manuscript.

Funding: This research was funded by the National Natural Science Foundation of China (grant nos. 11274397, 41176144, 41376139, and 41906109), the Government of Guangdong Province for the NST (grant no. 2016B090918099).

Institutional Review Board Statement: Not applicable.

Informed Consent Statement: Not applicable.

Data Availability Statement: Experimental data are available upon reasonable request to the authors.

Acknowledgments: The authors thank X. Wang for discussion.

Conflicts of Interest: The authors declare no conflict of interest. 


\section{References}

1. Garcia-Pichel, F. Cyanobacteria. Encyclopedia of Microbiology, 3rd ed.; Moselio, S., Ed.; Academic Press: San Diego, CA, USA, 2009; pp. 107-124.

2. Glazer, A.N. Light harvesting by phycobilisomes. Annu. Rev. Biophys. Biophys. Chem. 1985, 14, 47-77. [CrossRef]

3. Liu, L.; Chen, X.; Zhang, Y.; Zhou, B. Characterization, structure and function of linker polypeptides in phycobilisomes of cyanobacteria and red algae: An overview. Biochim. Biophys. Acta 2005, 1708, 133-142. [CrossRef]

4. Tian, L.; Gwezdala, M.; van Stokkum, I.H.M.; Koehorst, R.B.M.; Kirilovsky, D.; van Amerongen, H. Picosecond kinetics of light harvesting and photoprotective quenching in wild-type and mutant phycobilisomes isolated from the cyanobacterium synechocystis PCC 6803. Biophys. J. 2012, 102, 1692-1700. [CrossRef] [PubMed]

5. Falamas, A.; Porav, S.A.; Tosa, V. Investigations of the energy transfer in the phycobilisome antenna of arthrospira platensis using femtosecond spectroscopy. Appl. Sci. 2020, 10, 4045. [CrossRef]

6. Kolodny, Y.; Zer, H.; Propper, M.; Yochelis, S.; Paltiel, Y.; Keren, N. Marine cyanobacteria tune energy transfer efficiency in their light-harvesting antennae by modifying pigment coupling. FEBS. J. 2021, 288, 980-994. [CrossRef] [PubMed]

7. Adir, N. Elucidation of the molecular structures of components of the phycobilisome: Reconstructing a giant. Photosynth. Res. 2005, 85, 15-32. [CrossRef] [PubMed]

8. Gantt, E. Phycobilisomes. Annu. Rev. Plant Physiol. 1981, 32, 327-347. [CrossRef]

9. David, L.; Marx, A.; Adir, N. High-resolution crystal structures of trimeric and rod phycocyanin. J. Mol. Biol. 2011, 405, 201-213. [CrossRef]

10. Schirmer, T.; Bode, W.; Huber, R.; Sidler, W.; Zuber, H. X-ray crystallographic structure of the light-harvesting biliprotein c-phycocyanin from the thermophilic cyanobacterium mastigocladus laminosus and its resemblance to globin structures. J. Mol. Biol. 1985, 184, 257-277. [CrossRef]

11. Berns, D.S.; MacColl, R. Phycocyanin in Physical Chemical Studies. Chem. Rev. 1989, 89, 807-825. [CrossRef]

12. Adir, N.; Dobrovetsky, Y.; Lerner, N. Structure of c-phycocyanin from the thermophilic cyanobacterium synechococcus vulcanus at 2.5 Å: Structural implications for thermal stability in phycobilisome sssembly. J. Mol. Biol. 2001, 313, 71-81. [CrossRef]

13. Brejc, K.; Ficner, R.; Huber, R.; Steinbacher, S. Isolation, crystallization, crystal structure analysis and refinement of allophycocyanin from the cyanobacterium spirulina platensis at $2.3 \AA$ resolution. J. Mol. Biol. 1995, 249, 424-440. [CrossRef] [PubMed]

14. Ying, L.; Xie, X. Fluorescence spectroscopy, exciton dynamics, and photochemistry of single allophycocyanin trimers. J. Phys. Chem. B 1998, 102, 10399-10409. [CrossRef]

15. McGregor, A.; Klartag, M.; David, L.; Adir, N. Allophycocyanin trimer stability and functionality are primarily due to polar enhanced hydrophobicity of the phycocyanobilin binding pocket. J. Mol. Biol. 2008, 384, 406-421. [CrossRef] [PubMed]

16. Wang, Q.; Moerner, W.E. Dissecting pigment architecture of individual photosynthetic antenna complexes in solution. Proc. Natl. Acad. Sci. USA 2015, 112, 13880-13885. [CrossRef]

17. Womick, J.M.; Moran, A.M. Exciton coherence and energy transport in the light-harvesting dimers of allophycocyanin. J. Phys. Chem. B 2009, 113, 15747-15959. [CrossRef]

18. Zhang, J.; Zhao, J.; Jiang, L.; Zheng, X.; Zhao, F.; Wang, H. Studies on the energy transfer among the rod-core complex from phycobilisome of Anabaena variabilis by time resolved fluorescence emission and anisotropy spectra. Biochim. Biophys. Acta Bioener. 1997, 1320, 285-296. [CrossRef]

19. Hirota, Y.; Serikawa, H.; Kawakami, K.; Ueno, M.; Kamiya, N.; Kosumi, D. Ultrafast energy transfer dynamics of phycobilisome from Thermosynechococcus vulcanus, as revealed by ps fluorescence and fs pump-probe spectroscopies. Photosynth. Res. 2021, 148, 181-190. [CrossRef]

20. Adir, N.; Lerner, N. The crystal structure of a novel unmethylated form of c-phycocyanin, a possible connector between cores and rods in phycobilisomes. J. Biol. Chem. 2003, 278, 25926-25932. [CrossRef]

21. Marx, A.; Adir, N. Allophycocyanin and phycocyanin crystal structures reveal facets of phycobilisome assembly. Biochim. Biophys. Acta Bioener. 2013, 1827, 311-318. [CrossRef]

22. David, L.; Prado, M.; Arteni, A.; Elmlund, D.; Blankenship, R.; Adir, N. Structural studies show energy transfer within stabilized phycobilisomes independent of the mode of rod-core assembly. Biochim. Biophys. Acta 2014, 1837, 385-395. [CrossRef] [PubMed]

23. Adir, N.; Dines, M.; Klartag, M.; McGregor, A.; Melamed-Frank, M. Assembly and Disassembly of Phycobilisomes. In Complex Intracellular Structures in Prokaryotes. Microbiology Monographs, 1st ed.; Shively, J.M., Ed.; Springer: Berlin/Heidelberg, Germany, 2006; Volume 2, pp. 47-77.

24. Ma, J.; You, X.; Sun, S.; Wang, X.; Qin, S.; Sui, S. Structural basis of energy transfer in Porphyridium purpureum phycobilisome. Nature 2020, 579, 146-151. [CrossRef] [PubMed]

25. Wang, H.; Zhao, J.; Jiang, L. Energy transfer processes in phycobilisome model complex at 77 K. China Ser. B-Chem. 2000, 43, 233-239. [CrossRef]

26. Zhao, F.; Zheng, X.; Zhang, J.; Wang, H.; Yu, Z.; Zhao, J.; Jiang, L. Energy transfer in allophycocyanin hexamer from Anabaena variabilis by time-resolved spectroscopy. J. Photochem. Photobiol. B 1998, 45, 144-149. [CrossRef]

27. Sandstrom, A.; Gillbro, T.; Sandstrom, U.; Fischer, R.; Scheer, H. Picosecond study of energy transfer within 18-S particles of AN 112 (a mutant of Synechococcus 6301) phycobilisomes. Biochim. Biophys. Acta Bioener. 1988, 933, 54-64. [CrossRef]

28. Zhang, J.; Zhao, F.; Zheng, X.; Wang, H. Direct measurement of excitation transfer dynamics between two trimers in C-phycocyanin hexamer from cyanobacterium Anabaena variabilis. Chem. Phys. Lett. 1999, 304, 357-364. [CrossRef] 
29. Zhao, F.; Zhang, J.; Zheng, X.; Wang, H. Energy transfer among proteins in the phycobilisome of red algae. II. Energy transfer in synthesized complex of R-PE/R-PC and R-PE/APC. Zhongshan Da Xue Xue Bao. Zi Ran Ke Xue Ban Acta Sci. Nat. Univ. Sunyatseni 1998, 37, 18-23.

30. Holzwarth, A.R.; Wendler, J.; Suter, G.W. Studies on chromophore coupling in isolated phycobiliproteins: II. Picosecond energy transfer kinetics and time-resolved fluorescence spectra of C-phycocyanin from synechococcus 6301 as a function of the aggregation state. Biophys. J. 1987, 51, 1-12. [CrossRef] 